Objectives: To compare the epidemiological, clinical, biological, radiological, bacteriological profile as well as the management and prognosis of spondylodiscitis over the last 20 years.

Methods: Retrospective study including 113 patients hospitalized in our department between 1999 and 2018. The diagnosis was based on clinical, biological, radiological and bacteriological data. Spondylodiscitis were divided into two groups: spondylodiscitis occurring between 1998 and 2008 ( $1^{\text {st }}$ decade) and those between 2009 and 2018 ( $2^{\text {nd }}$ decade).

Results: Of the 113 identified patients, 54 (47.8\%) occurred between 1998 and 2008 , with male predominance in both groups $(50.2$ and $59.3 \%$ respectively; $p=0.3)$. The average age was 55 years $(p=0.7)$. Diabetes was more frequent in the $1^{\text {st }}$ decade but without a statistically significant difference $(\mathrm{p}=0.1)$. On the other hand, consumption of unpasteurized dairy products was more frequent during the $2^{\text {nd }}$ decade $(p<0.001)$ as well as the presence of intercurrent infection $(p=0.01)$.

The estimated delay between symptom onset and diagnosis was longer between 1998 and 2008 (5.6 months and 3.2 months respectively, $p=0.005$ ). The presence of neurological signs was more frequent between 2009 and $2018(p=0.001)$, especially radiculalgia $(p=0.02)$. The sedimentation rate was higher in the $1^{\text {st }}$ decade (93mm and $72 \mathrm{~mm}$ respectively, $\mathrm{p}=0.002$ ).

We found no statistically significant difference in the location of spondylodiscitis $(p=0.4)$ and the multifocal involvement $(p=0.5)$.

Radiographic signs were significantly more prevalent in the $1^{\text {st }}$ decade $(p=0.002)$, in particular disc space narrowing $(p=0.03)$ and irregularity of the intervertebral plates $(p=0.01)$. Computed tomography was more often performed during the $1^{\text {st }}$ decade $(p=0.008)$, unlike magnetic resonance imaging, performed in $88.1 \%$ of cases during the $2^{\text {nd }}$ decade $(p<0.001)$. In contrast, the frequency of para-vertebral abscesses, epiduritis and spinal cord compressions were similar in the two groups ( $p=0.6 ; p=0.9$ and $p=0.3$, respectively).

Tuberculosis was more frequent in the $1^{\text {st }}$ decade $(p=0.009)$. Disco-vertebral biopsy was more often performed in the $1^{\text {st }}$ decade $(p=0.04)$, but its efficiency was similar between the two groups $(p=0.1)$. For pyogenic spondylodiscitis, blood cultures were more often positive in the $2^{\text {nd }}$ decade but without a statistically significant difference $(p=0.6)$. On the other hand, the anatomopathological aspect was more frequently suggestive of a pyogenic germ in the $2^{\text {nd }}$ decade $(p=0.04)$.

A surgical procedure was more frequently performed between 2009 and 2018 but with no statistically significant difference $(p=0.2)$. All patients received antibiotic therapy. A change in treatment was made in $23.7 \%$ of cases between 2009 and $2018(p=0.01)$. The prognosis was better during the $1^{\text {st }}$ decade $(p=0.01)$. On the other hand, the occurrence of immediate complication during tuberculous spondylodiscitis was more frequent during the $2^{\text {nd }}$ decade $(p=0.03)$, in particular the toxicity of anti-tuberculosis treatment $(p=0.04)$.

Conclusion: In the last decade, the diagnosis of spondylodiscitis has been made earlier. However, these are more severe forms with an increased frequency of neurological complications, probably due to an increase in the proportion of virulent germs. Moreover, the effectiveness of the bacteriological investigation does not differ between the two periods studied.

Disclosure of Interests: None declared

DOI: 10.1136/annrheumdis-2020-eular.4814

\begin{tabular}{|l|l}
\hline FRI0434 & PREDICTIVE FACTORS OF SERIOUS INFECTIONS \\
& IN PATIENTS WITH CHRONIC INFLAMMATORY \\
& ARTHRITIS ON TREATMENT WITH TNF INHIBITORS: \\
& REAL LIFE DATA
\end{tabular}

A. Ruiz Ochoa ${ }^{1}$, J. Escudero Argaluza ${ }^{2}$, B. Alvarez Rodríguez ${ }^{2}$, M. Vasques Rocha $^{2}$, C. Stoye ${ }^{2}$, O. Pompei Fernández ${ }^{2}$, S. Gil ${ }^{2}$, J. R. De Dios ${ }^{2}$, J. CalvoAlen $^{2} .{ }^{1}$ Universidad del País Vasco, Vitoria, Spain; ${ }^{2}$ Hospital Universitario Araba, Rheumatology, Vitoria, Spain

Background: Serious infections are one of the most feared adverse events in patients treated with biologics. To this regard data coming from randomized and long-term extension studies may not totally applied to the usual clinical practice due to the different profile of the treated patients. To study the associated factors for serious infections in patients with inflammatory arthritis treated with TNF inhibitors

Objectives: To study the associated factors for serious infections in patients with inflammatory arthritis treated with TNF inhibitors

Methods: All the medical records of the patients with inflammatory arthritis being treated with TNF inhibitors at the beginning of 2016 were retrospectively reviewed. All serious infections suffered for these patients until the end of 2018 were recorded. Serious infections were defined as those which required to admitted at the hospital for intravenous treatment. Potential variables associated with the development of these infections including: demographic and clinical characteristics, concomitant treatments or comorbidity (by Charlson index) were studied. Standard statistical tests for descriptive and univariate analyses were used and a multivariable logistic regression model was built to check independent associations.

Results: Overall 334 patients (50.3\% women) with a mean age of 56.67 ( \pm 12.853$)$ were studied: 140 (41.92\%) Rheumatoid arthritis (RA), 55 (16.46\%) psoriatic arthritis (PsA) and $138(41.62 \%)$ spondyloarthritis (Sp). Forty-five serious infections were observed in 30 patients, being respiratory (40\%) and urinary $(8.8 \%)$ the most frequent localizations. Only one patient died. By univariate analysis, disease duration, age, concomitan use of glucocorticoids (GC) (but not of synthetic DMARDs), Charlson index and specifically Diabetes Mellitus were associated with infection $(p<0.05)$. The type of arthritis was not associated and the results in the subset of RA patients were overall similar. In the multivariate analysis the use of GC [OR: 5.31 (1.98.14.26)] and the Charlson index [OR:2.48 (1.70;3.60)] were found to be independently associated to infection.

Conclusion: In patients with inflammatory arthritis and treated with TNF inhibitors around a $10 \%$ developed any serious infection along three years of follow up. Use of GC and comorbidity emerged as the main risk factors for this complication.

Disclosure of Interests: None declared

DOI: 10.1136/annrheumdis-2020-eular.4347

\section{FRI0435 AUTOANTIBODIES AND HLA-B27 MOLECULE TESTING IN UYGUR PATIENTS WITH HUMAN IMMUNODEFICIENCY VIRUS INFECTION}

X. Chen ${ }^{1}$, L. Wu ${ }^{2}$, X. Wu ${ }^{2}$, C. N. Luo ${ }^{2}$, Y. M. Shi ${ }^{2} .{ }^{1}$ People's Hospital of Xinjiang Uygur Autonomous Region, Department of Rheumatology and Immunology, Urumqi, China; ${ }^{2}$ People's Hospital of Xinjiang Uygur Autonomous Region, Urumqi, China

Background: AIDS is a deadly infectious disease caused by the HIV. When HIV infects a host, it may induce production of autoantibodies due to the structural antigen similarity between viral proteins and selfantigens. The molecular mimicry between HIV protein and self-antigens could cause antibody cross-reactions and lead to development of autoimmune disease.

Objectives: To explore the clinical value of serum autoantibodies and human leukocyte antigen (HLA-B27) molecular testing in Uygur patients with human immunodeficiency virus (HIV) infection.

Methods: A total of 727 HIV-infected Uygur patients who visited Kuche Infectious Diseases Hospital during May 2016 to March 2017 were include in this study. The other 390 healthy people were enrolled as controls. Serum antinuclear antibodies (ANA) and ANA Profile, anti-cyclic citrullinated peptide (CCP) antibody, and HLA-B27 molecule were tested.

Results: Among 727 HIV-infected Uygur patients, 317 were males and 410 were females with mean age $(35.52 \pm 13.44)$ years old. The mean duration of disease was $(6.34 \pm 3.05)$ years. There were $697(95.87 \%)$ patients receiving Highly active antiretroviral therapy (HAART) with mean duration of treatment $(6.34 \pm 3.05)$ years. Rheumatic manifestations were recorded in 238 (32.74\%) HIV-infected Uygur patients, mainly with dry mouth and dry eye (15.41\%), alopecia $(9.90 \%)$, arthralgia $(8.94 \%)$, ect. Compared with the health controls, positive ANA was more common in HIV infected Uygur patients (33.42\% vs. $17.43 \%$, $P<0.001$ ) with low titers (ANA titer:1:100). HIV-infected Uygur patients had higher positive anti-u1-RNP antibodies positive rate (1.10\%), but lower anti-SSA antibodies positive rate $(0.14 \%)$ and anti-CCP antibodies positive rate $(0.28 \%)$. Patients with positive ANA in HAART group were significantly less than that in non-treatment group $(38.72 \%$ vs. $50.00 \%, P=0.049)$.Only one female patien was HLA-B27 positive $(0.14 \%)$, which was significantly lower than that in healthy controls $(3.08 \%)(P<0.001)$. Also, only one patient was diagnosed with rheumatoid arthritis (RA).

Conclusion: Rheumatic manifestations are common in HIV-infected Uygur patients. Several autoantibodies are positive, but the coincidence of rheumatic diseases is rare. It's noted that patients with Rheumatic manifestations and low titre positive ANA should be considered as a differential diagnosis of HIV infection.

Disclosure of Interests: None declared

DOI: 10.1136/annrheumdis-2020-eular.5155

\section{FRI0436 \\ ARTICULAR AND PERIARTICULAR INFECTIOUS DISEASES: REVIEW AND ANALYSIS OF A COHORT IN A 3RD LEVEL HOSPITAL}

R. Dos Santos Sobrín ${ }^{1}$, N. Pérez Gómez ${ }^{1}$, E. Perez-Pampín ${ }^{1}$, A. Souto Vilas ${ }^{1}$, A. Mera Varela ${ }^{1}{ }^{1}$ Clinical University Hospital in Santiago de Compostela, Rheumatology Department, Santiago de Compostela, Spain

Background: Infectious diseases in Rheumatology are still a prevalent healthcare issue nowadays. Microbiological tests and early beginning of antibiotic 
treatment are basic supportive therapy for those patients. In some cases, surgery is needed and it can involve prognostic features.

Objectives: To analyze prognostic factors related to articular and periarticular infectious diseases.

Methods: Patients hospitalized between January and December 2019 in Rheumatology Service (CHUS) and diagnosed of articular or periarticular infectious disease were enrolled. Demographic, clinical and therapeutic data were collected.

Stata 15.1 was used to perform statistical analysis.

Results: 21 patients diagnosed of articular or periarticular infectious disease were included (138 patients hospitalized in 2019). Mean age was 57,3 years old (SD 18,2) and $76,2 \%$ were males. One cardiovascular risk factor was achieved, at least, by $71,4 \%$ (arterial hypertension the most frequent, followed by hypercholesterolemia and hyperuricemia). Gram positive cocci were identified in $76,2 \%$ of patients (Staphylococcus aureus in $47,6 \%$, only one case oxacillin-resistant). A concomitant rheumatic disease was present in 4 patients (2 suffer from gout). No statistical association was found between this topic and acute-phase reactants (APR), complication rates or days of hospitalization. At hospitalization time, mean values of erythrocyte sedimentation rate (ESR) were 65,6 mm (SD 30,8) and C-reactive protein (CRP) 11,7 mg/dl (SD $7,7)$. Both have a positive association with days of hospitalization $\left(r_{E S R}=0,282\right.$ $\left.r_{C R P}=0,424\right)$ and further, ESR demonstrates a positive correlation with this topic $(\mathrm{Cl} 95 \%-0,009$ to $0,723 \mathrm{p}=0,05569)$. Only 3 patients needed adjuvant surgery (articular infectious diseases with higher APR values). Surgery demonstrates a relationship statistically significant with articular involvement $(t=-3,72 p=0,00)$, higher hospitalization rates $(t=-2,51 p=0,02)$ and complication rates $(\mathrm{Chi} 2=4,67 \mathrm{p}=0,03$ as decease, recurrence, bacteriemia, aftermath or soft tissue abscess).

Conclusion: Gout is the main rheumatic disease associated, in this study, to infectious diseases of musculoskeletal system. As opposed, to suffer a rheumatic disease isn't associate to a bad prognostic (hospitalization or complication rates). Furthermore, this cohort shows us the high prevalence of cardiovascular risk factors related with these diseases (hyperuricemia as predecessor of gout). Surgery intensifies as the main bad prognostic factor regarding days of hospitalization, complication rates and higher APR.

References:

[1] Morbimortality in adult patients with septic arthritis: a three-year hospital-based study. Ferrand J, et al. BMC Infect Dis 2016

[2] Predictors of treatment failure and mortality in native septic arthritis. Maneiro JR, et al. Clin Rheum 2014

Disclosure of Interests: None declared

DOI: 10.1136/annrheumdis-2020-eular.4807

\section{FRI0437 CT GUIDED NEEDLE BIOPSY IN VERTEBRAL OSTEOMYELITIS: RELIABILITY ANALYSIS AND STUDY OF WHICH FACTORS COULD INFLUENCE ON THE RESULT}

J. J. Fragio-Gil ${ }^{1}$, R. Gonzalez Mazario수, E. Grau García ${ }^{1}$, M. De la Rubia Navarro ${ }^{1}$, C. Pávez Perales ${ }^{1}$, S. Leal Rodriguez ${ }^{1}$, J. Ivorra Cortés ${ }^{1}$, L. Gonzalez Puig $^{1}$, R. Negueroles Albuixech ${ }^{1}$, I. Martínez Cordellat ${ }^{1}$, I. Chalmeta Verdejo ${ }^{1}$, J. E. Oller Rodríguez ${ }^{1}$, C. Alcañiz Escandell ${ }^{1}$, E. Vicens Bernabeu ${ }^{1}$, F. M. Ortiz Sanjuan ${ }^{1}$, C. Nájera Herranz ${ }^{1}$, I. Cánovas Olmos ${ }^{1}$, A. J. Cañada Martinez², M. Salavert Lleti ${ }^{3}$, J. A. Román Ivorra ${ }^{1}{ }^{1}$ Hospital Universitario y Politécnico La $\mathrm{Fe}$, Rheumatology, València, Spain; ${ }^{2}$ Instituto de Investigación Sanitaria La Fe de Valencia, Biostatistic, València, Spain; ${ }^{3}$ Hospital Universitario y Politécnico de La Fe, Infectious Disease Unit, València, Spain

Background: Vertebral Osteomyelitis is an infectious disease of the vertebral body, also termed spondylodiscitis if the intervertebral disc is involved (which its avascular). Since the bacteriological characterization is in many times difficult and blood cultures are often negative, a bone biopsy is in most of the cases encouraged.

Objectives: The aim of this study is to analyze which factors could influence on the result of a CT guided biopsy (CTGB) in vertebral spondylodiscitis patients.

Methods: A retrospective observational study was performed including patients diagnosed of spondylodiscitis in a single center who underwent a CTGB. Demographic features and comorbidities, acute phase markers, microbiological results, radiological data, antibiotic exposure, medical complications and the clinical outcomes were also collected for analysis. Standard procedure in our center is performed by Musculoskeletal Specialized Radiologist under local anesthesia and CT control. Abscess sample is collected with a $18 \mathrm{G}$ needle with coaxial technique, trying to obtain at least 3 samples. For discal space, a thicker needle (13.5G-15G) is used. A logistic regression including cofounding factors was performed using $\mathrm{R}$ software.
Results: A total of 86 were included with a mean age of 62.75 (14.98) years old and predominationg male sex (68.60\%). 15 patients (17.44\%) presented any kind of immunosuppression. Clinical data are summarized in Table 1. Blood cultures were positive in $39.71 \%$ and sample culture showed a reliability of $49 \%$. Organism which grew were gram $+(66.67 \%)$, gram $-(12.70 \%)$, mycobacteria $(12.7 \%)$ and fungi $(7.94 \%)$. In only 16 cases $(18.6 \%)$ there was isolated the same organism in blood and on biopsy culture. From admission to procedure, a mean of 6 days was observed. Antibiotic treatment had a median value of 2 days $(0,6)$ and its exposure did not modified the culture positivity (IC 95\% [0.2745.211] $p=0.816$ ). Detailed analysis was performed looking for the influence of the days of exposure, which also failed (IC 95\% [0.939-1.101] $p=0.747$ ). The longer duration of the pain was related to a higher probability of obtaining a negative result on the biopsy (IC 95\% [1.004-1.035] $p=0.026$ ) (graphic 1). Neither fever $(p=0.303)$ or higher CRP (IC 95\% [0.992-1.006] $p=0.761)$ value modified the culture result.

Table 1. Demographic and clinical characteristics.

\begin{tabular}{lcc}
\hline & $\mathrm{N}=86$ & $\%$ \\
\hline Clinical history & & \\
High blood pressure & 42 & 48.84 \\
Diabetes Mellitus & 19 & 22.09 \\
Liver cirrhosis & 16 & 18.60 \\
Chronic kidney failure & 13 & 15.12 \\
Active Systemic Malignancy* & 2 & 2.33 \\
Rheumatoid arthritis* & 3 & 3.49 \\
Spondyloarthritis* & 1 & 1.16 \\
HIV infection* & 4 & 4.65 \\
Solid organ transplant receptor* & 3 & $3.49 \%$ \\
Systemic Amyloidosis* & 1 & 1.16 \\
Splenectomy* & 2 & 2.33 \\
Previous spine pathology & 50 & 58.14 \\
Underlying/associated endocarditis & 2 & $2.33 \%$ \\
& &
\end{tabular}

${ }^{*}$ Considered as immunosuppressed patients

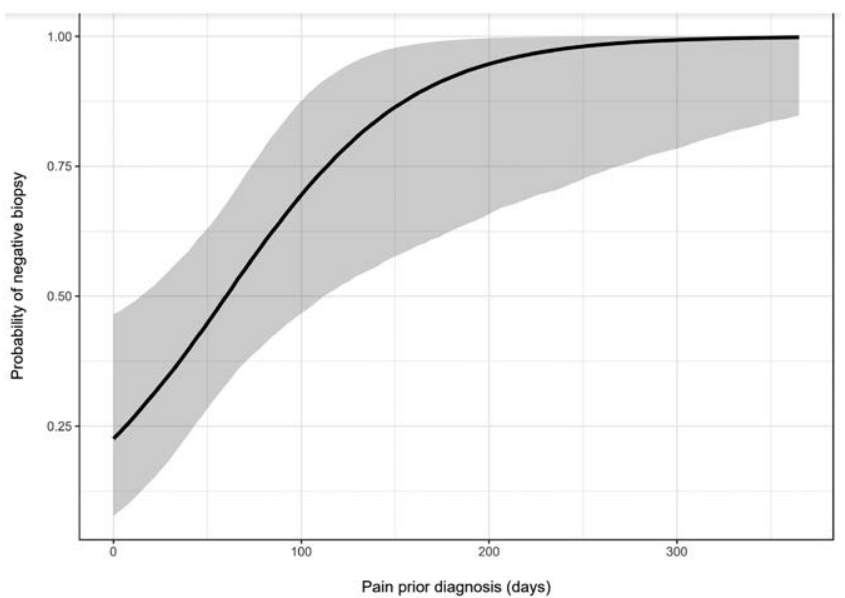

Conclusion: Even in cases under antibiotic treatment, CTGB displays an acceptable reliability. The longer the length of painful period before diagnosis was related to a higher chance of obtaining a negative result on culture. This result could be explained by a greater aggressiveness of pyogenic organisms that perhaps congregate in the lesser time span instead of non-pyogenic agents, that could deliver in more silent infection.

References:

[1] IDSA Clinical Practice Guidelines for the Diagnosis and Treatment of Native Vertebral Osteomyelitis in Adults

Disclosure of Interests: None declared

DOI: 10.1136/annrheumdis-2020-eular.6383

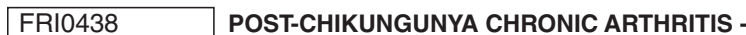 SHARING OF BANGLADESH EXPERIENCE OVER ONE} YEAR FOLLOW UP OF 60 PATIENTS

S. Hossain ${ }^{1}$, M. Choudhury², M. A. Islam², M. M. Hassan², S. Yeasmin²,

F. Hossain ${ }^{3} .{ }^{1}$ Bangabandhu Sheikh Mujib Medical University (BSMMU), Department of Rheumatology, Dhaka, Bangladesh; ${ }^{1}$ Bangabandhu Sheikh Mujib Medical University (BSMMU), Department of Rheumatology, Dhaka, Bangladesh; ${ }^{3}$ Freelance Researcher, Dhaka, Bangladesh 